\title{
IOT Application of a Remote Weather Monitoring \& Surveillance Station
}

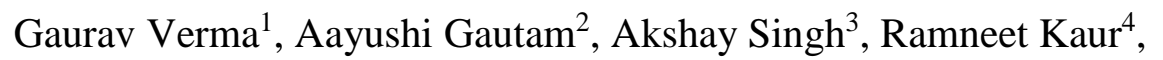 \\ Apoorva Garg ${ }^{5}$ and Manya Mehta ${ }^{6}$ \\ Department of Electronics \& Communication Engineering, \\ 1,2,4,5 Jaypee Institute of Information Technology, A-10, Sector-62, Noida (U.P.), \\ India \\ ${ }^{4}$ Amity University, Sector-125, Noida, (U.P.), India
}

\begin{abstract}
Numerous critical weather occasions have influenced humanity throughout the years. For quite a while climate checking was to a great extent a leisure activity of eager beginners. Yet in the course of the most recent century, it has advanced into an efficient and expert worldwide action that mirrors its vital significance for an extensive variety of economic, natural, civil assurance and farming exercises. Present day climate observing frameworks and systems are intended to be developed in a financially savvy way. This requires the aggregate life-cycle cost of an observing framework is minimized, and one approach to do this is to minimize the maintenance of the weather monitoring framework. Utilizing a solid-state system to quantify the weather, including the wind speed and bearing, is principal to minimize hardware adjusting and costs. The conventional weather monitoring framework comprised of individual sensors to quantify one meteorological variable, each associated with an data collection gadget or recorder. Modern day innovation has permitted the combination of a few sensors into one coordinated weather station that can be for all time situated at one site, or transported to a site where localized climate is required. The fundamental aim of this paper is to design a remote weather monitoring system which allows the monitoring of weather parameters and provides continuous surveillance at the same time. IOT based Remote Weather Monitoring Station is a fully-fledged open source weather station which is effective in measuring temperature, humidity, and light intensity with high precision and the values of measured parameters are plotted on open cloud "ThingSpeak". The system is also equipped with a camera to provide the live streaming of the area to be monitored. The controlling action is accomplished using an Embedded- PC (Raspberry Pi) or Arduino board with WIFI extension interface. In this paper the weather station is currently controlled using Raspberry Pi board and programming is done in python language.
\end{abstract}

Keywords: Internet of things, Putty, Raspberry Pi, ThingSpeak, Remote monitoring, Weather Station

\section{Introduction}

The Internet of Things (IoT) is the grid of physical objects or "things" beneath which is present electronics, software, sensors, and network connectivity that allows these objects or things to exchange data or communicate "Things" in IoT can be any manmade or artificial object that can be provided with an IP address so that they can communicate. Weather is made and changed by the air, particularly the troposphere, which is in persistent motion. The troposphere is the layer of the environment that is nearest to Earth. It is around 10 kilometers inside and out, where it meets the stratosphere. It is for the most part noted on an everyday premise, with thoughtfulness regarding specific day by day changes in the examples that bring precipitation, high winds, frosty temperatures etc. 
Weather predictions are made for a day, two days or up to a week ahead of time and this helps us settle on our day by day choices about heading out and how to dress for the climate. So we can make an idea of about how important is the weather monitoring task. Since time immemorial various technologies have been used for the monitoring and surveillance purpose. One such technology is RADAR. In the past half century, climate radar has progressed incredibly and has helped made progress in a wide range of meteorological and climatologically applications. Of specific significance has been its capacity to identify and caution of hazards connected with extreme local hailstorms, tornadoes, high winds, and extraordinary precipitation. Today, climate radars enhance aviation wellbeing and increment the operational proficiency of the whole air transport industry, and they add to farming cautions and flood notices through checking of precipitation intensity.

In our research we talk about a different technology that make use of IoT for better results and efficient weather monitoring in Real time. Various IoT based systems contain pair of sensors connected through a Raspberry Pi (ARMv6) microcontroller which measures variables like temperature and humidity through a DHT11 sensor. With the help of sensors data is collected and then using microcontroller it is sent to the cloud. All temperature and humidity data is logged at user-defined intervals for tracking trends over time, reporting capabilities, and at-a-glance monitoring to help users make informed decisions. Stationary Weather Stations serve as a disadvantage, being positioned to measure the temperature values of a single location only. To overcome this disadvantage, the weather station has been made mobile i.e., it has been mounted upon a 3-wheeled bot which can be internet controlled through Raspberry Pi. The system is based on IoT hence, enables user to check weather data online from any place anywhere. The data is plotted as temp-humidity curve, as histograms which makes observation easier.

\section{Literature Survey}

The Internet of Things (IoT) is the new wave of development that guarantees to enhance and improve our day to day life in view of smart sensors and objects working in a well coordinated manner. Through Internet Protocol (IP) availability, devices can now be associated with the Internet, in this manner permitting them to be managed and controlled wherever and whenever. [1] Gives a review of the endeavors in the Internet Engineering Task Force (IETF) to standardize security answers for the IoT biological system. It give a top to bottom survey of the communication security solutions for IoT, particularly the standard security conventions to be utilized in conjunction with the Constrained Application Protocol (CoAP), an application protocol particularly designed to adjust to the constraints of IoT devices. It also additionally talks about the most recent standardization endeavors to adjust and improve the DTLS for IoT applications. Numerous Internet of Things (IoT) advances have been utilized for money flow, logistics flow, people flow etc. To deal with these expanding dissimilar devices and network alternatives, [3] ETSI has determined end-to-end machine-to-machine (M2M) framework design for IoT applications. In light of this design, an IoT EasyConnect framework is built to deal with IoT devices. In this approach, an IoT device is described by its "elements" (e.g., temperature, vibration, and show) that are controlled by the system applications. Other technologies have also formed the basis of the design required to implement the system. Recent advancements have reformed the embedded technology in a way that each client carries a smart device (robots) which makes their life less demanding and agreeable. In [8] a self-governing portable robot used for remote position following utilizing GPS and sending that data on to a device, for example, versatile or tablet utilizing GSM is developed. This robot consist of GPS (for identification of area), GSM (for remote information exchange), DTMF (for controlling robot with versatile or tab), sonar sensor (for obstruction discovery) and glimmer light (for night vision). This 
portable robot has many applications in protection, aviation, horticulture, and security etc. When we talk about system design it is important to emphasize on security of the data which is transmitted. [9] Concentrates on SSL which is a procedure used to give customer and server validation, information classification and information trustworthiness. It changes the information into ambiguous shape, information which will be transmitted can be in text form or any other form, by encoding the information it is saved from eavesdropping of any other unauthenticated attack. This system of secure information transmission is extremely valuable in securing the integrity of information sent by the Unmanned Aerial Vehicles in military application to financially utilized Electricity meter. Since the previously mentioned gadgets utilizes microcontroller to send information through web thus this information is continually going to face previously mentioned dangers. Thus managing security issues turns out to be major task.

\section{Proposed Work}

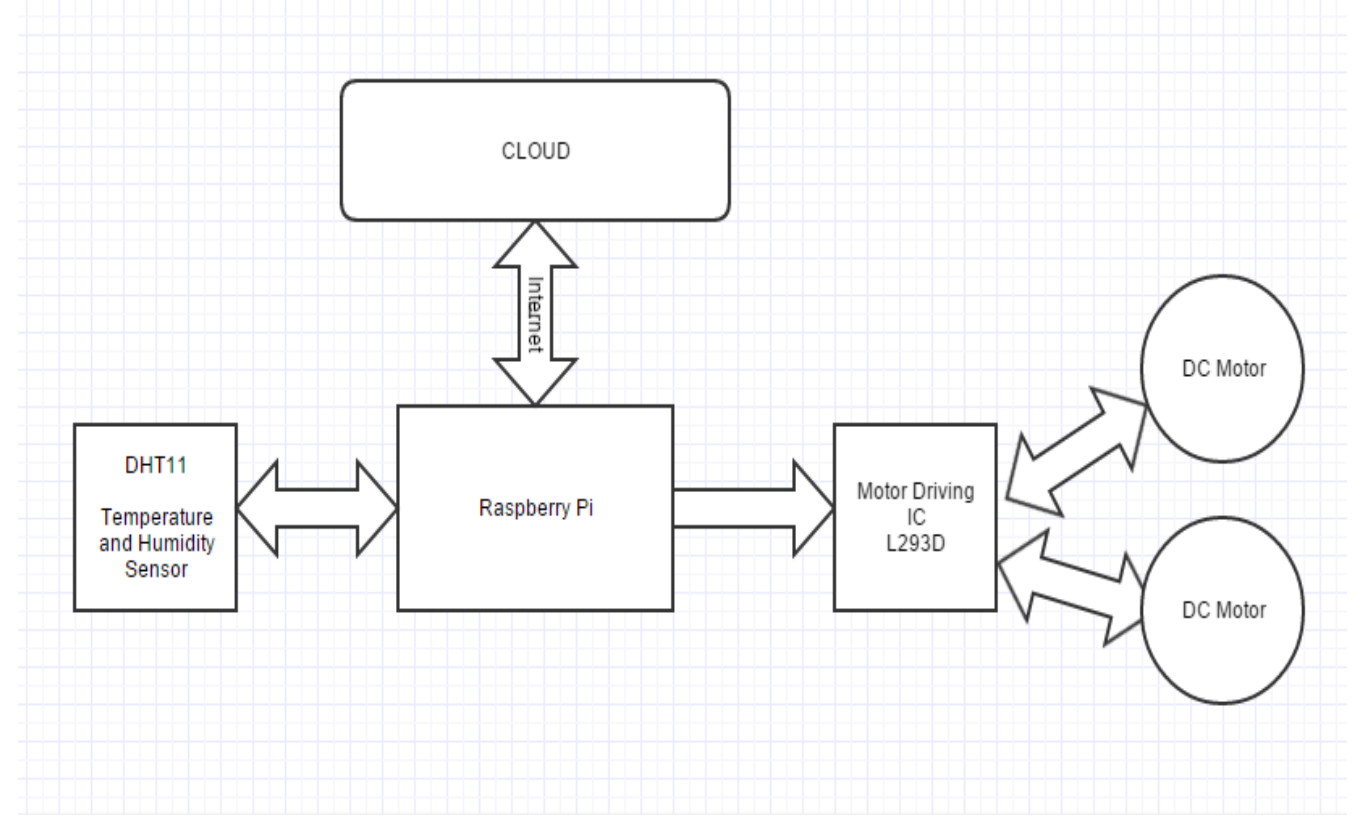

Figure 1. Block Diagram of the System

The 3 wheeled bot is made of 2 DC motors, which can be rotated in both forward and reverse directions using an H-Bridge. L293D IC serves as the H-Bridge. Raspberry Pi has been used as the microcontroller in the project which is using LINUX as its OS. The coding has been done using Python version 2.7. To connect to the raspberry pi, PuTTY has been used as a terminal client for secure SSH login and operations over the Raspberry Pi.

DHT11 is a temperature and humidity sensor, it takes the value of temperature in Celsius. The data is then uploaded to the internet through the wifi interface. The user can make an account on www.thingspeak.com, which can record and log the temperature and humidity values and help in plotting graphs and curves of the data so that the data can be analyzed in an easier manner. Client have (MCU) to send a flag, DHT11 changed over from low-control mode to rapid mode, until the host started to flag the end of the DHT11 send a reaction flag to send 40bit information, and trigger a letter gathering. The flag is sent as appears in Figure 2. 


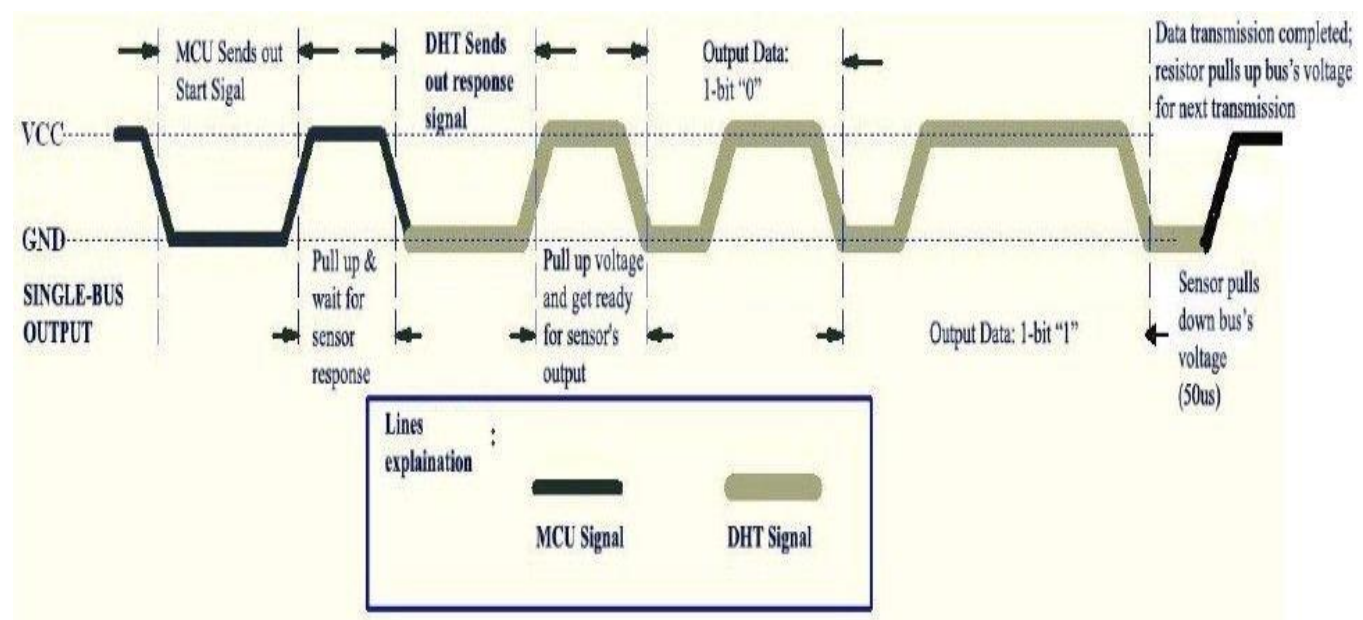

Figure 2. Data Timing Diagram

\section{A. ThingSpeak (Cloud)}

ThingSpeak is an open source Internet of Things application and API to store and recover information from things utilizing the HTTP convention over the Internet or by means of a Local Area Network. ThingSpeak empowers the production of sensor logging applications, area following applications, and an interpersonal organization of things with announcements. ThingSpeak has support from numerical processing programming MATLAB from MathWorks. [4] Its having a facility of real-time data collection, MATLAB support and open API.

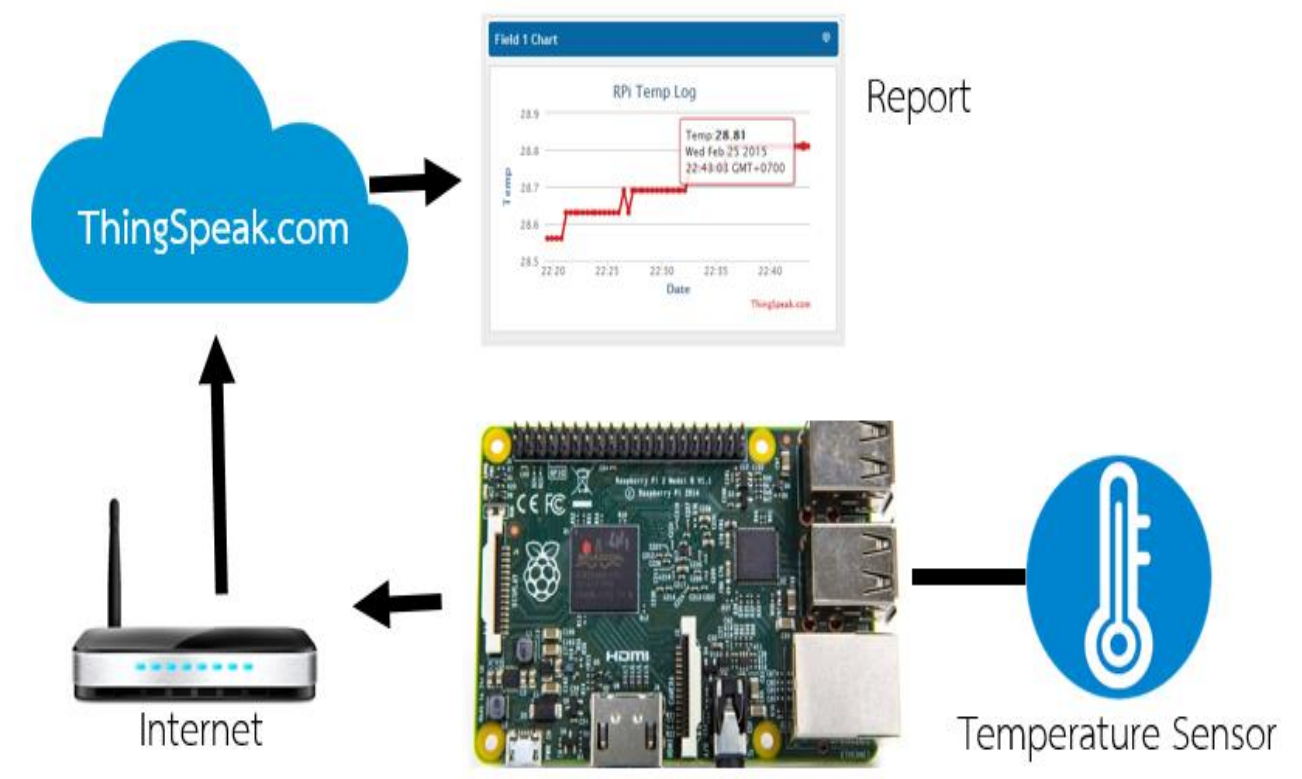

Figure 3. ThingSpeak-How it Works [4]

\section{B. PUTTY Software}

PuTTY is a free and open-source terminal emulator; serial reassure and system record exchange application. It supports a few system conventions, including SCP, SSH, Telnet, rlogin, and crude attachment association. It can likewise interface with a serial port. PuTTY supports numerous minor variations from the safe remote terminal, and gives client control over the SSH encryption key and convention protocol, exchange figures i.e., 
ciphers for example, 3DES, Arcfour, Blowfish, DES, and Public-key confirmation. It can likewise copy control groupings from xterm, VT102 or ECMA-48 terminal imitating, and permits nearby, remote, or element port forwarding with SSH (counting X11 sending). The system correspondence layer underpins IPv6, and the SSH convention supports the zlib@openssh.com which is a delayed compression scheme. It can likewise be utilized with neighborhood serial port associations. PuTTY comes packaged with command line SCP and SFTP customers, called "pscp" and "psftp" individually.

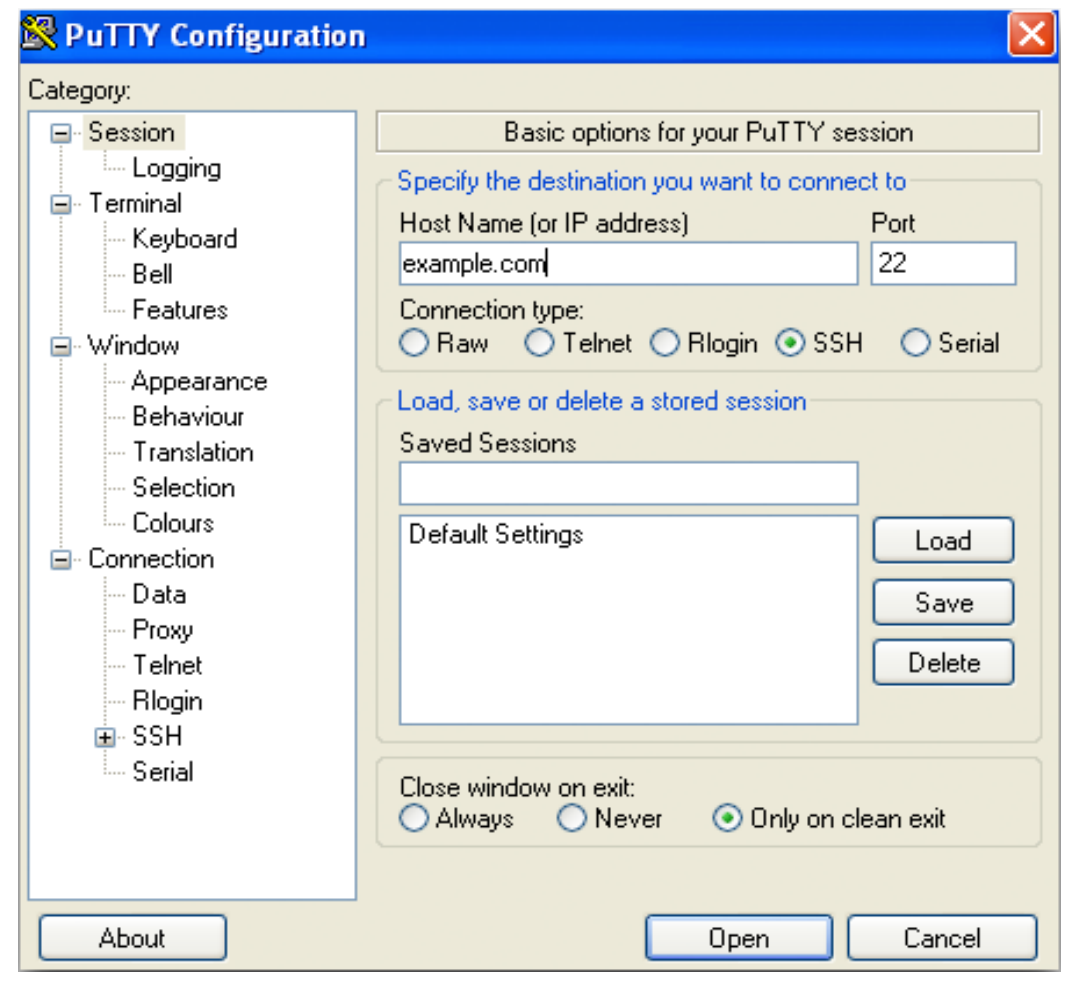

Figure 4. PuTTY Launch Screen

\section{Fritzing Software}

Fritzing is an open source software initiative to support designers ready to move from physical prototyping to actual product. Fritzing can be seen as an electronic design automation (EDA) tool for engineers. The associated website helps users share and discuss drafts and experiences as well as to reduce manufacturing costs: [5] 


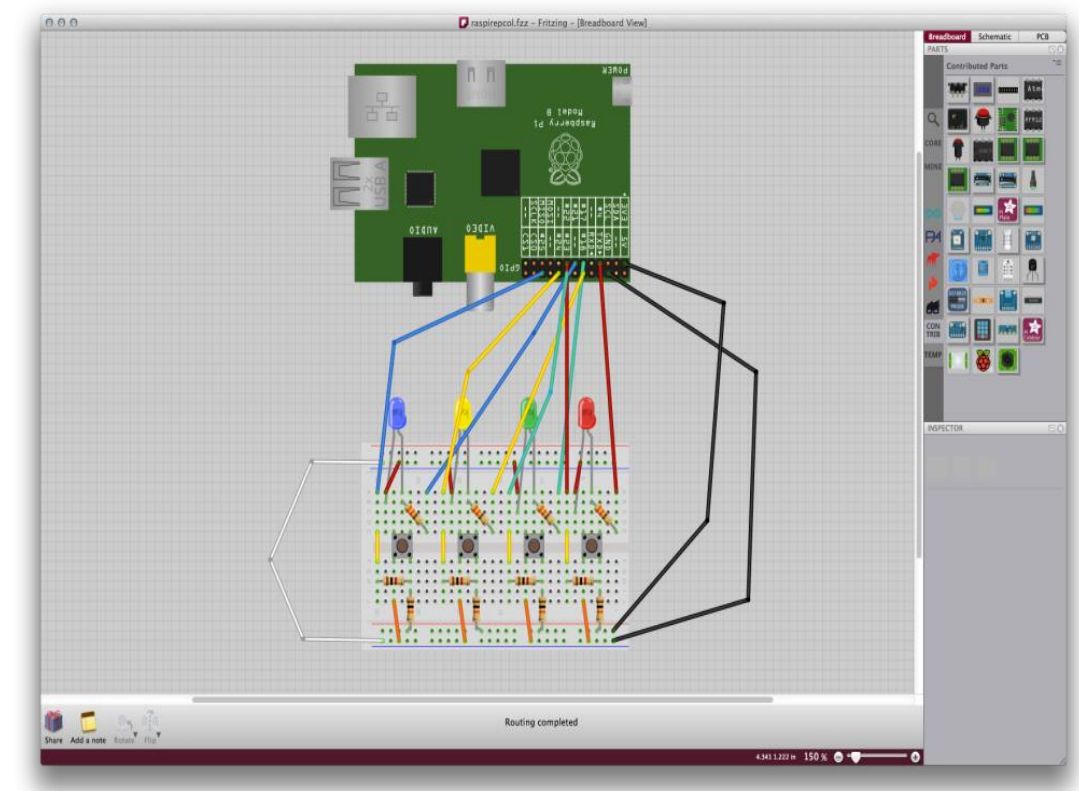

Figure 5. Fritzing Breadboard View [5]

\section{Hardware Implementation}

The hardware part has 3 major parts: A Raspberry Pi, Temperature sensor DHT11, and motor driving IC-L293D.

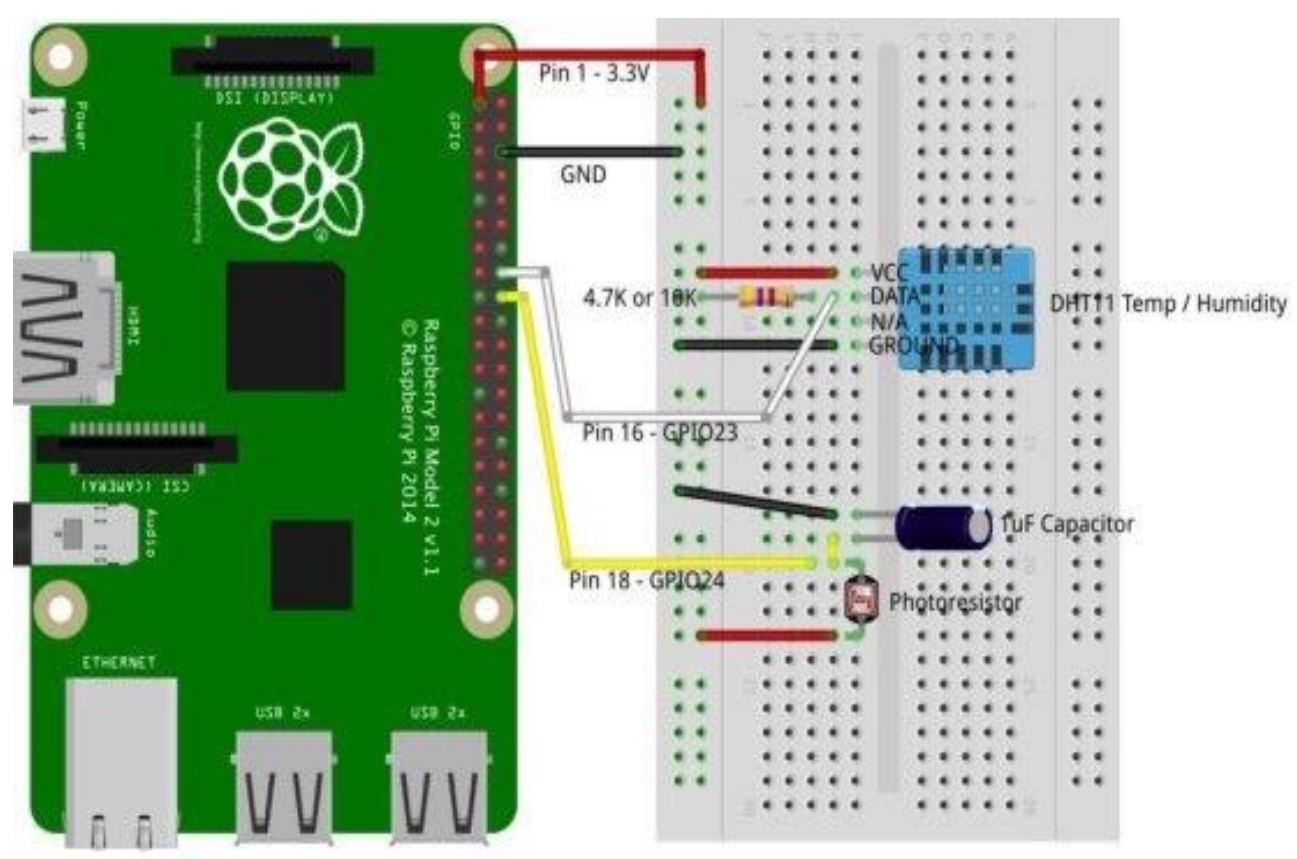

fritzing

Figure 6. Interfacing of DHT11 with Pi [5] 
Table 1.1-DHT11 Connections with Pi

\begin{tabular}{|c|c|}
\hline DHT11 & Raspberry Pi \\
\hline VCC & 3V3 Pin 1 \\
\hline Data & GPIO 23 or Pin16 \\
\hline Ground & GND Pin 18 \\
\hline
\end{tabular}

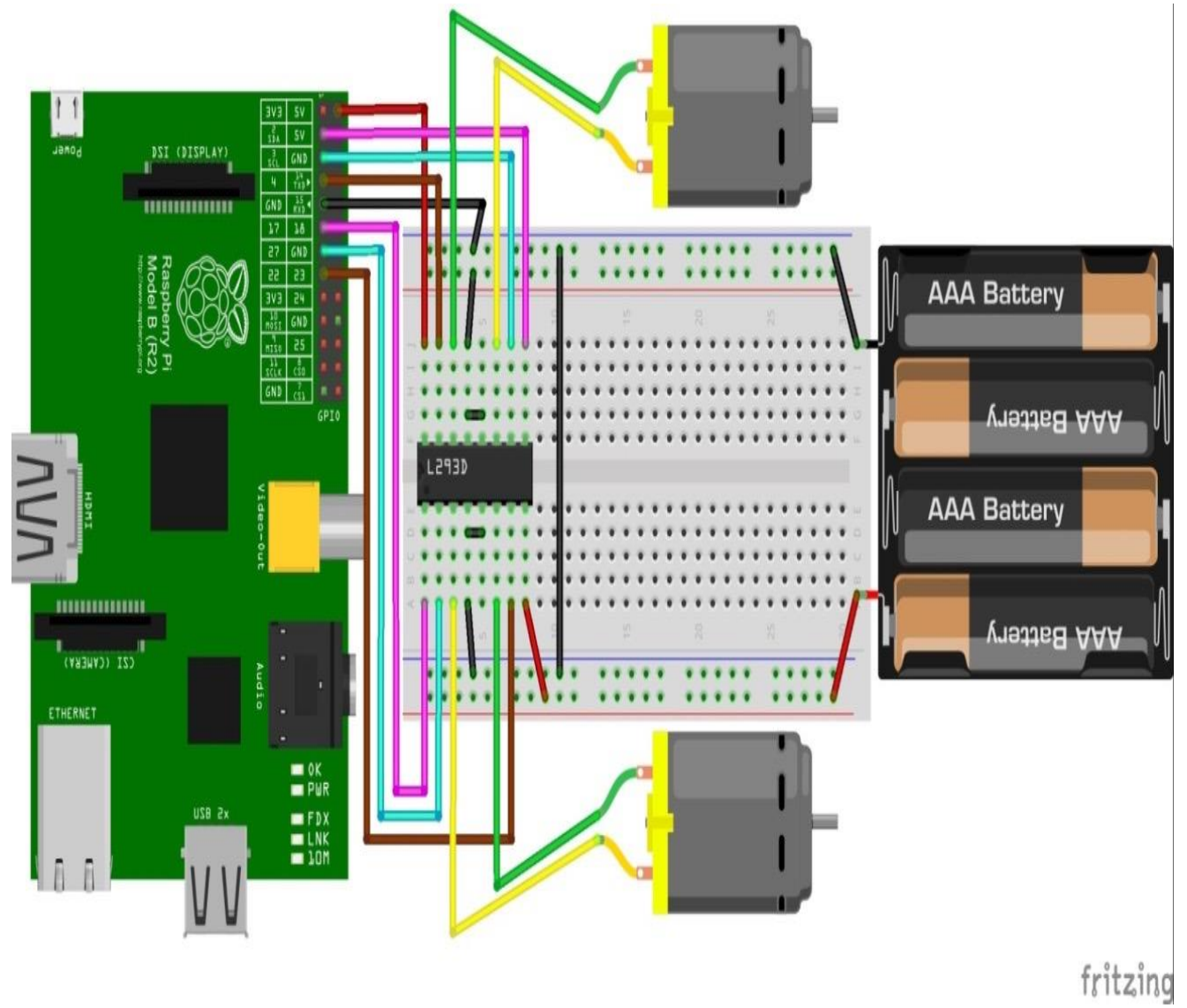

Figure 7. L293D Interfacing with Pi [5]

There are 4 input pins for 1293d, stick 2, 7 on the left and stick 15, 10 as shown in the pin diagram. Left input pins will control the turn of engine associated crosswise over left side and right input for engine on the right hand side. The engines are pivoted on the basis of the sources of input given over the pins as LOGIC 0 or LOGIC 1 . VCC is the voltage that it requirements for its own particular internal operation 5v; L293D won't utilize this voltage for driving the engine. For driving the engines it has a different arrangement to give engine supply VSS (V supply). L293D will utilize this to drive the engine. It implies in the event that we need to work an engine at $9 \mathrm{~V}$ then you have to give a Supply of $9 \mathrm{~V}$ crosswise over VSS Motor supply.

\section{Results and Analysis}

The following are the results of temperature and humidity data plotted on our ThingSpeak channel and the picture of final working model as shown in Figure 8 and 9: 




Field 2 Chart

是

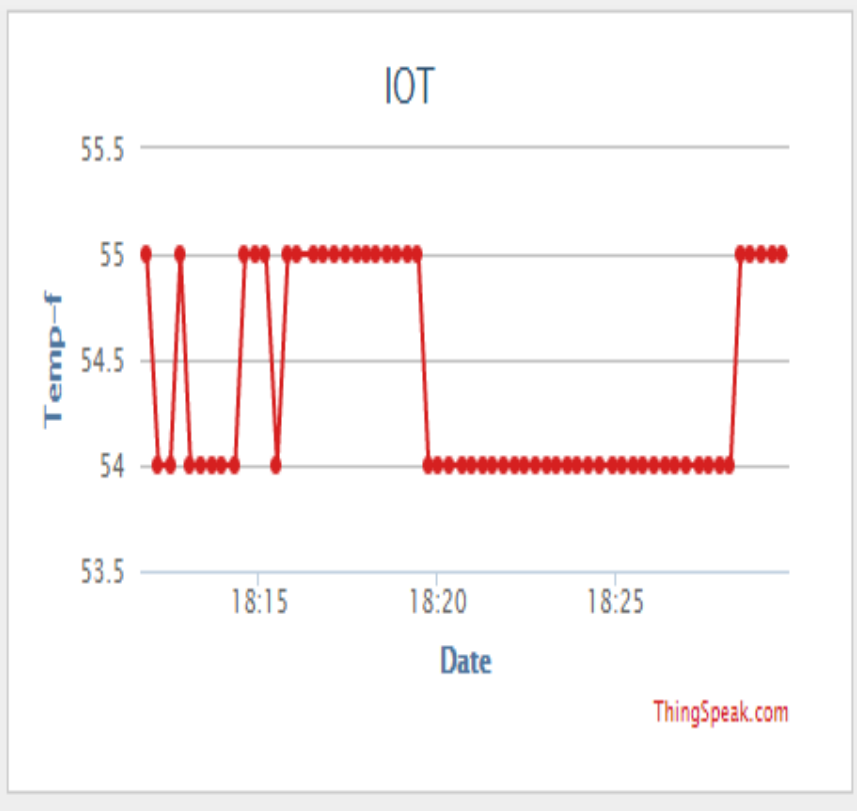

Figure 8. Temperature Data Plot ${ }^{[4]}$ 


\section{Field 3 Chart}

星

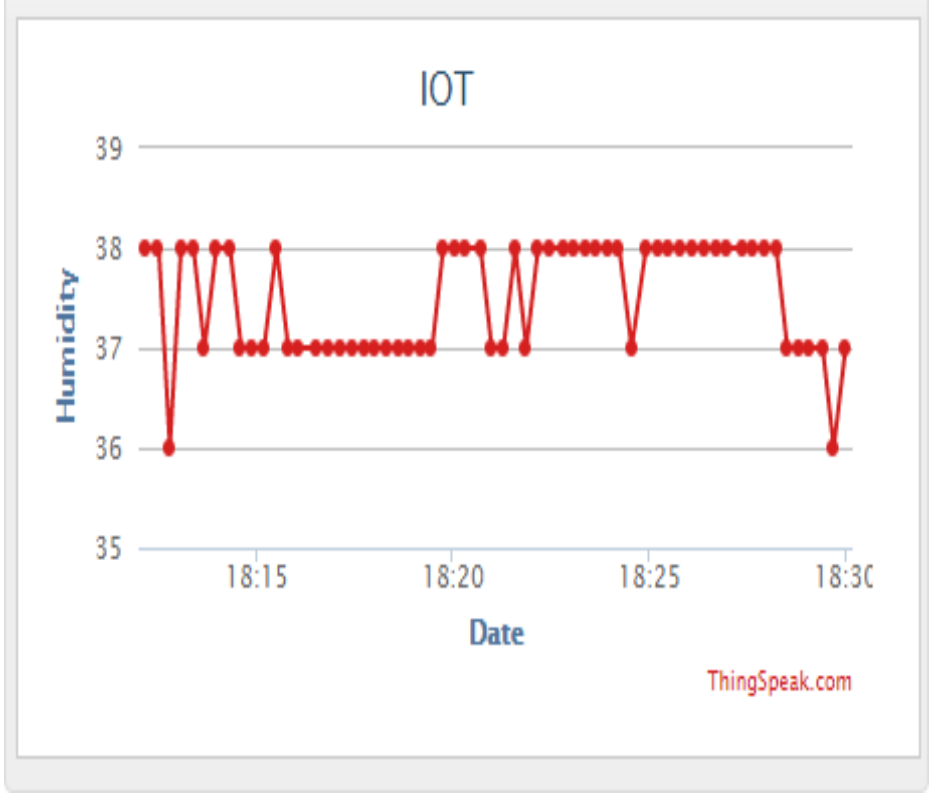

Figure 9. Humidity Data Plot [4]

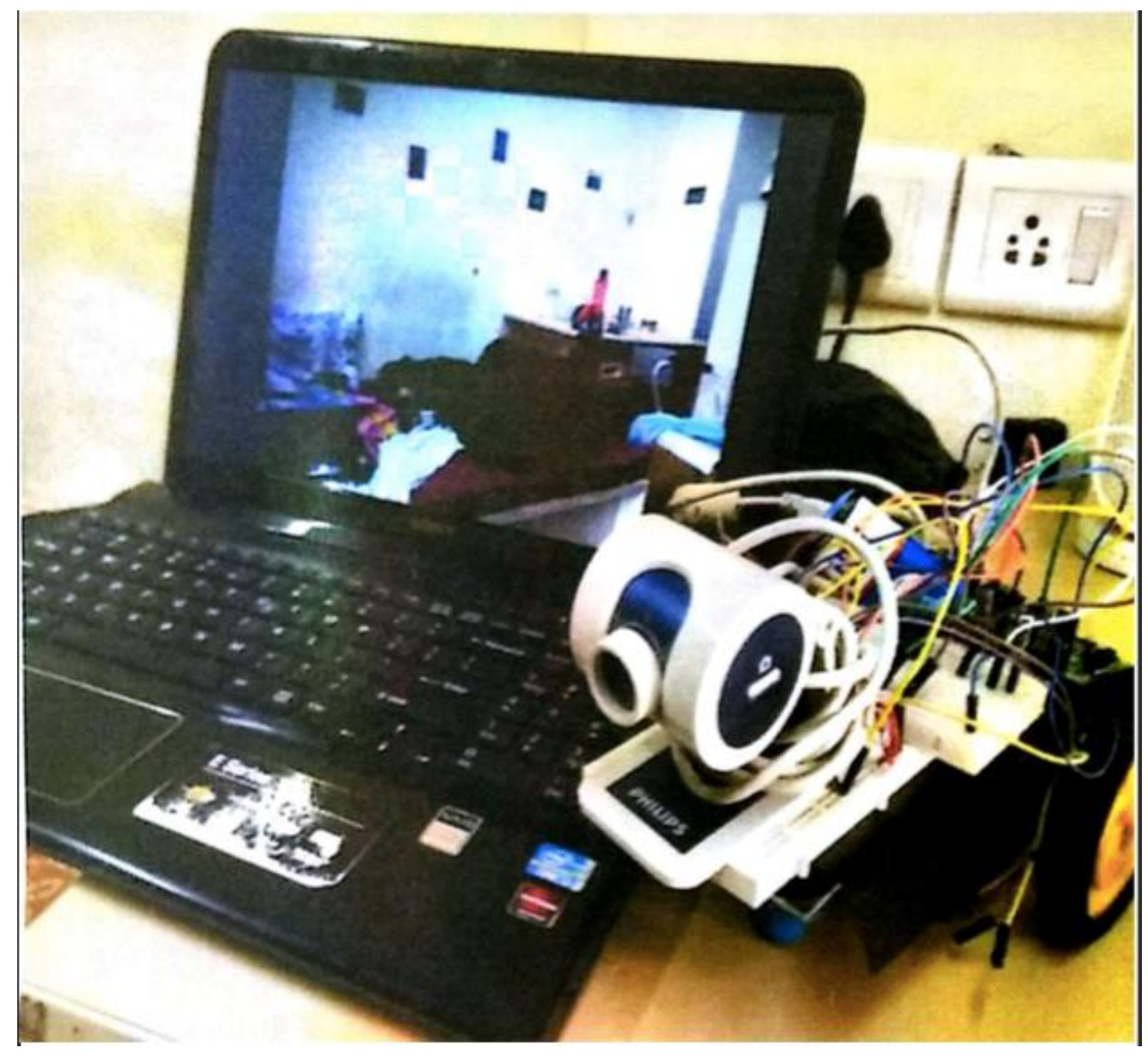

Figure 10. Working Test Model 


\section{Conclusion}

There are numerous advantages of consolidating IoT into our lives, which can help people, organizations, and society every day. For people this new idea can come in numerous structures including well being, security, monetarily, and consistently arranging. IoT helps in better control over asset usage. The internet of things could likewise help clients guarantee the usage of their hardware and offices is at 100 percent or as near it as would be prudent, minimize downtime, and recognize and address any bottle necks or under-use to maximize throughput. In worldwide endeavors, this framework could give an information stream that outcomes in a thorough perspective of the profitability and execution of hardware, permitting clients to write about the data diverse criteria. Associated sensors could likewise supports disaster administration, provoking moment activity to act when levels of required parameter fall beneath or over a specific level.

\section{Future Work}

This can be implemented as a part of a larger space exploration robot with more sensors like Fire sensor, Gas sensor etc. Also, weather forecasting using tools like Regression can be an option.

\section{References}

[1] SyeLoongKeoh and S. S. Kumar, "Securing the Internet of Things: A Standardization Perspective", Internet of Things Journal, vol. 1, Issue 3, (2004) June.

[2] J. Pan, R. Jain and S. Paul, "An Internet of Things Framework for Smart Energy in Buildings: Designs, Prototype, and Experiments", Internet of Things Journal, IEEE vol. 2, Issue: 6, (2015) October.

[3] Y.-B. Lin, Y.-W. Lin and C.-Y. Chih, "EasyConnect: A Management System for IoT Devices and Its Applications for Interactive Design and Art", Internet of Things Journal, IEEE vol. 2, Issue: 6, (2015) October.

[4] ThingSpeakhttp://thingspeak.com/

[5] Fritzinghttp://fritzing.org/home/

[6] S. Jambhulkar, A. Vikram, S. K. Virdi, P. Sharma, K. Goel and G. Verma, "Android Application Based Mishap Identification and Warning System", International Journal of Control and Application, vol. 9, no. 8, (2016) September, pp. 41-48.

[7] A. Gautam, D. Bareja, S. K. Virdi, S. Shekar and G. Verma, "Implementation of High Performance Home Automation using Arduino", Indian Journal of Science and Technology, vol. 9, issue 21, (2016) June.

[8] G. Verma, H. Verma, I. Singh, A. Vikram, S. Singhal, A. Kumar, S. Banarwal and K. Goel, "Wireless Position Tracking of a DTMF based Mobile Robot using GSM and GPS", Indian Journal of Science and Technology", vol. 8, issue 17, IPL0161, (2015) August.

[9] V. Negi, H. Verma, I. Singh, A. Vikram, K. Malik, A. Singh and G. Verma, "Network Security in Embedded System Using TLS", International Journal of Security \& its Applications, vol. 10, no. 2, pp. 375-384, (2016) February.

[10] G. Verma, V. Verma, S. Jhambhulkar and H. Verma, "Design of a Lead-Lag Compensator for Position Loop Control of a Gimballed Payload", in Proceedings of IEEE International Conference on Signal Processing and Integrated Networks (SPIN-2015), (2015) February 19-20, pp. 394-399. 